\title{
Aszites, Pfortaderthrombose und hepatische Enzephalopathie bei Leberzirrhose: Aktuelle Therapieempfehlungen
}

\author{
Alexander L. Gerbes ${ }^{a, b}$ Veit Gülberg ${ }^{b, c}$ \\ ${ }^{\text {a }}$ Medizinische Klinik und Poliklinik II, \\ ${ }^{\text {b } L e b e r-C e n t r u m ~ M u ̈ n c h e n ~}{ }^{\circledR}$, \\ ${ }^{\mathrm{c}}$ Medizinische Klinik und Poliklinik IV, Klinikum der Universität, Ludwig-Maximilians-Universität, München, Deutschland
}

\section{Schlüsselwörter}

Aszites · Pfortaderthrombose .

Hepatische Enzephalopathie · Leberzirrhose

\section{Zusammenfassung}

Hintergrund: Aszites, Pfortaderthrombose und hepatische Enzephalopathie sind wichtige Komplikationen bei einer Leberzirrhose. Zur Therapie des Aszites gibt es aktuelle Leitlinien der DGVS. Methode: Das Manuskript fasst aktuelle Empfehlungen auf Basis der S3-Leitlinie der DGVS sowie anderer Leitlinien und aus Sicht der Autoren zusammen. Ergebnisse und Schlussfolgerungen: Bei fehlenden Kontraindikationen ist die TIPS(transjuguläre intrahepatische portosystemische Shunt)-Anlage das Verfahren der Wahl bei therapierefraktärem oder rezidivierendem Aszites. Für das hepatorenale Syndrom Typ 1 ist die Wirksamkeit einer Therapie mit Albumin und dem Vasokonstriktor Terlipressin belegt. Bei der Pfortaderthrombose wird häufig eine therapeutische Eskalation bestehend aus Antikoagulation, TIPS und Lebertransplantation verfolgt. Die Suche nach den Auslösern und eine entsprechende Therapie ist die wichtigste therapeutische Strategie bei hepatischer Enzephalopathie. Rifaximin hat einen zunehmenden Stellenwert in der medikamentösen Therapie und Rezidivprophylaxe der hepatischen Enzephalopathie.

\author{
Keywords \\ Ascites - Portal vein thrombosis . \\ Hepatic encephalopathy · Cirrhosis of the liver

\section{Summary \\ Treatment of Ascites, Portal Vein Thrombosis and Hepatic Encephalopathy in Patients with Cirrhosis of the Liver}

Background: Ascites, portal vein thrombosis and hepatic encephalopathy are important complications of cirrhosis of the liver. Guidelines for the treatment of ascites have recently been published. Method: This manuscript summarizes up-to-date recommendations on the basis of the DGVS S3 guideline and of other guidelines as well as of the authors' experience. Results and Conclusions: TIPS (transjugular intrahepatic portosystemic shunt) is the preferred treatment for refractory or recidivant ascites unless there are contraindications. The therapy of hepatorenal syndrome type 1 with albumin and the vasoconstrictor Terlipressin has been proven effective. Treatment of portal vein thrombosis comprises a strategy of anticoagulation, TIPS and liver transplantation. The most important therapeutic strategy for hepatic encephalopathy is the search for as well as the treatment of trigger events. Rifaximin is being increasingly used for the treatment and prophylaxis of hepatic encephalopathy.

\section{KARGER \\ Fax +497614520714 \\ Information@Karger.de}

www.karger.com (c) 2012 S. Karger GmbH, Freiburg

1662-6664/12/0285-0297\$38.00/0

Accessible online at:

www.karger.com/vim
Prof. Dr. med. Alexander Gerbes

Medizinische Klinik und Poliklinik II / Leber-Centrum München

Klinikum der Ludwig-Maximilians-Universität München

Marchioninistraße 15, 81377 München, Deutschland

Gerbes@med.uni-muenchen.de 


\section{Aszites}

\section{Therapie des Aszites}

Zur Therapie des Aszites sind unlängst Empfehlungen im Rahmen einer S3-Leitlinie der Deutschen Gesellschaft für Verdauungs- und Stoffwechselkrankheiten (DGVS) veröffentlicht worden [1]. Die weiteren Ausführungen beziehen sich überwiegend auf diese Leitlinie. Für die Pathophysiologie der Aszitesbildung spielt die Verminderung des effektiven Blutvolumens bei Leberzirrhose eine wesentliche Rolle (Abb. 1). Konventionelle und neue Therapieverfahren sind in Bezug auf diese pathophysiologische Besonderheit zu sehen.

\section{Medikamentöse Therapie des Aszites}

Mehrere Studien untersuchten das neuere Schleifendiuretikum Torasemid in seiner Wirkung bei Patienten mit Leberzirrhose und Aszites [2-4]. Torasemid zeigte sich hierbei als mindestens gleich effektiv und sicher wie Furosemid und kann somit als Alternative zu Furosemid angesehen werden [2-4] (Kasten 1).

Bei Patienten, die nicht ausreichend auf die diuretische Therapie ansprechen, sollte die Natriumexkretion im 24-Stunden-Sammelurin gemessen werden, um echte Nonresponder von Patienten zu unterscheiden, die keine Kochsalzrestriktion einhalten. Bei einer 24-Stunden-Natriumausscheidung über $85 \mathrm{mmol}$ ohne Gewichtsverlust ist davon auszugehen, dass die Kochsalzaufnahme über $5 \mathrm{~g} /$ Tag liegt.

\section{Definitionen des therapierefraktären und rezidivierenden Aszites}

Der therapierefraktäre Aszites ist als diuretikaresistenter oder intraktabler Aszites definiert [5]. Diuretikaresistenz bedeutet inadäquates Ansprechen auf Natriumrestriktion und eine hoch dosierte diuretische Therapie (Spironolacton maximal 400 mg/Tag und Furosemid maximal 160 mg/Tag). Beim intraktablen Aszites bestehen Komplikationen wie hepatische Enzephalopathie oder ausgeprägte Hyponatriämie, die eine adäquate diuretische Therapie verhindern. Rezidivierender Aszites ist definiert als Aszites, der trotz Natriumrestriktion und Verschreibung von Diuretika in adäquater Dosierung mindestens dreimal in einem Zeitraum von $1 \mathrm{Jahr}$ wieder auftritt (Kasten 2).

Abgesehen von der Lebertransplantation, die bei therapierefraktärem oder intraktablem Aszites immer erwogen werden sollte, stehen die wiederholte großvolumige Parazentese und der transjuguläre intrahepatische portosystemische Shunt (TIPS) als therapeutische Alternativen zur Verfügung. Die Anlage eines peritoneovenösen Shunts ist mit einem sehr

Hämodynamische Veränderungen

Portale Hypertension, periphere Vasodilatation Vermindertes effektives Blutvolumen

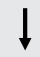

Neurohumorale Gegenregulation

Renin-Aldosteron, Sympathikus, ADH

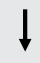

Beeinträchtigung der Nierenfunktion

Retention von $\mathrm{Na}^{+}$und $\mathrm{H}_{2} \mathrm{O}$, Vasokonstriktion

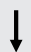

Aszites, Niereninsuffizienz, Hepatorenales Syndrom

Abb. 1. Funktionelles Nierenversagen, Aszites und HRS bei Leberzirrhose.

Kasten 1. Empfehlungen zur medikamentösen Therapie des Aszites (nach [1]).

\footnotetext{
- Als Diuretikum der ersten Wahl soll der orale Aldosteronantagonist Spironolacton eingesetzt werden. Die Initialdosis beträgt in der Regel 100 mg/Tag.

- Bei unzureichender Aszitesmobilisation sollte die Medikation mit einem Schleifendiuretikum ergänzt werden.

- Bei Patienten mit ausgeprägtem oder länger bestehendem Aszites sollte initial eine Kombinationstherapie aus Spironolacton und einem Schleifendiuretikum erwogen werden.

- Bei ausgeprägter Hyponatriämie ( $<125 \mathrm{mmol} / \mathrm{l})$, klinisch manifester hepatischer Enzephalopathie oder einer deutlichen Nierenfunktionsverschlechterung sollte auf Diuretika verzichtet werden.
}

Kasten 2. Empfehlungen zum therapierefraktären und rezidivierenden Aszites (nach [1]).

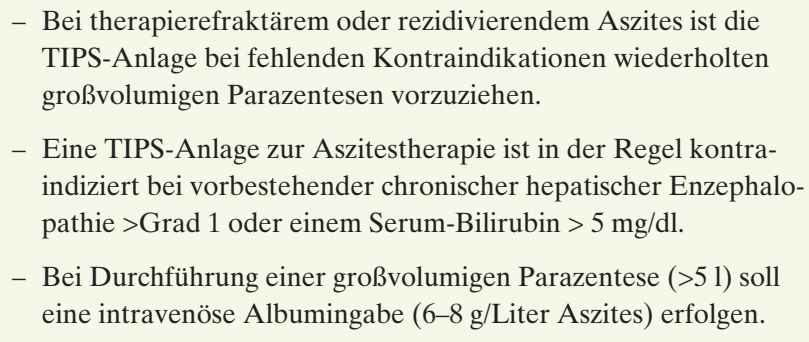
TIPS-Anlage bei fehlenden Kontraindikationen wiederholten großvolumigen Parazentesen vorzuziehen.

- Eine TIPS-Anlage zur Aszitestherapie ist in der Regel kontraindiziert bei vorbestehender chronischer hepatischer Enzephalopathie $>$ Grad 1 oder einem Serum-Bilirubin $>5 \mathrm{mg} / \mathrm{dl}$.

- Bei Durchführung einer großvolumigen Parazentese (>5 1) soll eine intravenöse Albumingabe (6-8 g/Liter Aszites) erfolgen.

hohen Komplikationsrisiko behaftet und dient lediglich in ausgewählten Einzelfällen noch als Reservefahren [6]. Eine subkutan zu implantierende Aszitespumpe könnte eine interessante Therapieoption darstellen; hierzu liegen erste Daten vor [7].

Die Rationale der TIPS-Anlage besteht darin, den portalen Druck und damit die Transsudation in die Peritonealhöhle $\mathrm{zu}$ reduzieren und das zentrale effektive Herzvolumen zu erhöhen. 4 Wochen nach TIPS-Anlage zeigen sich Natrium- 
ausscheidung und Nierenfunktion verbessert $[8,9]$. Weiterhin sinken die Plasma-Renin-Aktivität sowie die Aldosteron- und Noradrenalin-Konzentrationen. Dadurch werden die zirkulatorische Dysfunktion bei Leberzirrhose, die portale Hypertension und die Nierenfunktion verbessert.

Im Vergleich zur wiederholten großvolumigen Parazentese ist die TIPS-Anlage zur Aszitestherapie effektiver [10-14]. Bezüglich der Mortalität waren die Daten zunächst uneinheitlich. Die methodisch beste Metaanalyse auf der Basis individueller Patientendaten konnte allerdings eine niedrigere Mortalität nach TIPS im Vergleich zur Parazentese belegen [12]. Andere Komplikationen der portalen Hypertension, wie die gastrointestinale Blutung, die spontan bakterielle Peritonitis und das hepatorenale Syndrom (HRS), traten signifikant seltener nach TIPS-Anlage als bei regelmäßiger Parazentese auf [12]. Ein positiver Effekt der TIPS-Anlage auf die Lebensqualität wurde in einer Studie gezeigt [15].

\section{Hepatorenales Syndrom}

Das HRS ist definiert als potenziell reversible Nierenfunktionsstörung bei Patienten mit Leberzirrhose und Aszites oder bei Patienten mit alkoholischer Steatohepatitis. Es werden zwei Formen unterschieden [16]:

- HRS Typ I ist charakterisiert durch rasches Nierenversagen, definiert als Verdoppelung des Serumkreatinins auf über $2,5 \mathrm{mg} / \mathrm{dl}(226 \mathrm{mmol} / \mathrm{l})$ in weniger als 2 Wochen.

- HRS Typ II ist oft mit refraktärem Aszites vergesellschaftet und zeigt ein moderates Nierenversagen mit Serumkreatininwerten zwischen 1,5 und 2,5 mg/dl (133-226 mmol/l) bei stabilem oder langsam fortschreitendem Verlauf.

\section{Diagnostische Kriterien}

- Zirrhose mit Aszites (oder alkoholische Steatohepatitis)

- Serumkreatinin >1,5 mg/dl (>133 mmol/l)

- Keine Besserung des Serumkreatinins auf Werte $<1,5 \mathrm{mg} / \mathrm{dl}$ nach mindestens zweitägiger Pausierung aller Diuretika und Volumenexpansion mit Albumin. Die empfohlene Albumindosierung beträgt $1 \mathrm{~g} / \mathrm{kg}$ Körpergewicht pro Tag bis zu einem Maximum von $100 \mathrm{~g} / \mathrm{Tag}$.

- Ausschluss eines Schockgeschehens

- Keine laufende oder kürzlich erfolgte Therapie mit nephrotoxischen Medikamenten.

- Ausschluss einer parenchymatösen Nierenerkrankung (keine Proteinurie $>500 \mathrm{mg} / \mathrm{Tag}$ und unauffälliges Urinsediment und keine Mikrohämaturie >50 Erythrozyten/ HPF und unauffällige Nierensonographie).

In den letzten Jahren wurde die effektive Behandlungsmöglichkeit des HRS durch den Vasokonstriktor Terlipressin in Kombination mit Albumin gezeigt (Tab. 1, Kasten 3) [17].
Tab. 1. Terlipressin plus Albumin versus Albumin bei HRS (adaptiert nach [17])
Relatives Risiko

Nierenfunktion verbessert $\quad 3,76(2,21-6,39)$

Mortalität

$0,81(0,68-0,97)$

Nebenwirkungen $9,00(2,14-37,85)$

\section{Kasten 3.}

Empfehlungen zu den diagnostischen Kriterien des HRS (nach [1]).

Sofern keine Kontraindikationen vorliegen, sollen Patienten mit HRS Typ I mit Terlipressin und Albumin behandelt werden, da dies das kurzfristige Überleben signifikant verbessert.

- Diese Therapie soll mit einer Terlipressindosis von 2-4 mg/Tag begonnen werden und über einen Zeitraum von mindestens 3 Tagen durchgeführt werden. Terlipressin soll maximal in einer Dosis von 8-12 mg/Tag eingesetzt werden.

- Ein Nutzen dieser Therapie für Patienten mit HRS Typ II ist bislang nicht klar belegt; deswegen sollten diese Patienten analog zu Patienten mit refraktärem Aszites behandelt werden.

- Andere Vasokonstriktoren sind hinsichtlich Wirksamkeit, Überlebensvorteil und Nebenwirkungsprofil noch unzureichend untersucht und sollten daher nicht außerhalb von Studien eingesetzt werden.

Da Nierenersatzverfahren allein die Prognose bei HRS nicht verbessern, können sie bei Vorliegen von Dialysekriterien vor allem als Überbrückung bis zur Lebertransplantation eingesetzt werden.

Die extrakorporale Albumindialyse hat keinen Einfluss auf Nierenfunktion und Überleben bei Patienten mit HRS [18]. Leberersatzverfahren sollten daher derzeit bei Patienten mit HRS nur im Rahmen kontrollierter Studien zum Einsatz kommen.

\section{Pfortaderthrombose bei Leberzirrhose}

Pfortaderthrombosen finden sich meistens bei Leberzirrhose oder Malignomen der Leber [19, 20]. Im Stadium der kompensierten Zirrhose ist das Vorliegen einer Pfortaderthrombose äußerst ungewöhnlich, auch weil eine Thrombosierung meist zur Dekompensation führt. Mit zunehmender Schwere der Lebererkrankung steigt die Inzidenz auf $12-18$ pro 100 Patientenjahre [21, 22]. Hierfür scheint vor allem eine Reduktion der Fließgeschwindigkeit des portalen Blutstroms verantwortlich zu sein [23]. Wie bei Patienten mit Pfortaderthrombose ohne Lebererkrankung oder Malignom scheint jedoch das Vorliegen der Prothrombin-Mutation auch bei Leberzirrhose einen Risikofaktor darzustellen, der unabhängig von der Schwere der Lebererkrankung zur Thrombose prädisponiert [24]. 
Abb. 2a Die Farbkodierte Dopplersonographie (FKDS) bei einem Patienten mit dekompensierter Leberzirrhose zeigt eine subtotale Okklusion des Pfortaderhauptstamms (gelbe Markierung). b In der portalvenösen Phase des Abdomen-CT bei einem anderen Patienten findet sich eine vollständige Obstruktion des rechten Pfortaderasts mit Protrusion des Thrombus in die Pfortadergabel (weiße Pfeile).

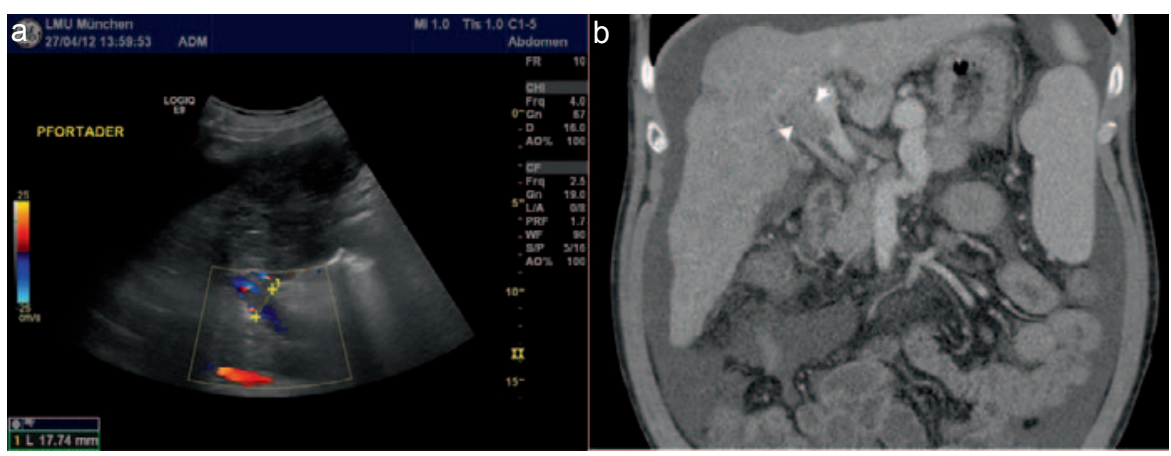

\section{Diagnostik}

Klinische Symptome einer Pfortaderthrombose sind meist unspezifisch oder fehlen völlig. Am häufigsten wird sie bei einer sonographischen oder CT-Untersuchung entdeckt, die im Rahmen der Überwachung auf ein mögliches hepatozelluläres Karzinom (HCC) oder wegen klinischer Verschlechterung mit hydropischer Dekompensation veranlasst wurden (Abb. 2).

Anders als bei Patienten mit Pfortaderthrombose ohne Zirrhose oder Tumorerkrankung ist der Nutzen eines Screenings auf hyperkoagulatorische Erkrankungen für Patienten mit Leberzirrhose nicht gesichert [25]. Eine neu aufgetretene Pfortaderthrombose bei Leberzirrhose sollte jedoch auf jeden Fall den Verdacht auf ein HCC nahelegen, weshalb die Bestimmung des Alpha-Fetoproteins (AFP) und die Durchführung einer dynamischen Bildgebung (kontrastverstärkter Ultraschall, CT oder MRT) mit arterieller und portalvenöser Phase sinnvoll erscheinen [26].

Zur Evaluation auf das Vorliegen blutungsgefährdeter gastroösophagealer Varizen sollte sowohl bei akuter als auch bei chronischer Pfortaderthrombose eine Endoskopie durchgeführt und gegebenenfalls eine primäre Blutungsprophylaxe eingeleitet werden. Hierzu kommen sowohl nichtselektive Betablocker als auch die endoskopische Gummibandligatur in Betracht [27].

\section{Therapie}

\section{Antikoagulation}

Der Stellenwert einer Antikoagulation bei Patienten mit Pfortaderthrombose und Leberzirrhose ist nicht definitiv geklärt. In einer unkontrollierten, prospektiven Studie an diesem Kollektiv konnte jedoch mit niedermolekularem Heparin (Enoxaparin $200 \mathrm{IU} / \mathrm{kg} / \mathrm{Tag}$ ) langfristig eine Rekanalisation der Pfortader bei $75 \%$ der 39 behandelten Patienten erreicht werden, ohne dass relevante Komplikationen - insbesondere Blutungen - beobachtet wurden [28]. Eine längerfristige, orale Antikoagulation mit Vitamin-K-Antagonisten muss sicherlich wegen der schwierigeren Steuerbarkeit bei Patienten mit Leberzirrhose kritisch diskutiert werden.
Tab. 2. Mögliche Auslöser einer hepatischen Enzephalopathie

\begin{tabular}{|c|c|}
\hline Erhöhte Ammoniakbelastung & $\begin{array}{l}\text { obere gastrointestinale Blutung } \\
\text { Obstipation } \\
\text { Nierenversagen } \\
\text { exzessive Proteinzufuhr } \\
\text { (insbesondere tierisches Eiweiß) }\end{array}$ \\
\hline Elektrolytentgleisung & $\begin{array}{l}\text { Hyponatriämie } \\
\text { Hypokaliämie } \\
\text { Dehydratation (Diuretika, Erbrechen, } \\
\text { Diarrhö) }\end{array}$ \\
\hline Medikamente & $\begin{array}{l}\text { Benzodiazepine } \\
\text { Opiate } \\
\text { Antihistaminika ( } \mathrm{H}_{1} \text {-Antagonisten) } \\
\text { Diuretika } \\
\text { sonstige Sedativa }\end{array}$ \\
\hline Verschiedenes & $\begin{array}{l}\text { bakterielle Infektionen } \\
\text { portosystemische Shunts (TIPS, } \\
\text { operative Shunts, spontane Shunts) }\end{array}$ \\
\hline
\end{tabular}

Transjugulärer intrahepatischer portosystemischer Shunt

Während ältere Leitlinien die TIPS-Anlage bei Vorliegen einer Pfortaderthrombose zumindest als relativ kontraindiziert einstuften, zeigen aktuelle Studien [29-31] für Patienten mit Leberzirrhose und Pfortaderthrombose vielversprechende Ergebnisse, insbesondere bei Vorliegen von weiteren Indikationen für einen TIPS - wie refraktären Aszites oder hepatischen Hydrothorax. Auch zur Prävention des Fortschreitens einer inkompletten Pfortaderthrombose wurde der TIPS bei Patienten auf der Transplantationswarteliste erfolgreich eingesetzt [32]. Sicherlich macht das Vorliegen einer partiellen oder kompletten Pfortaderthrombose die Anlage eines TIPS technisch anspruchsvoller, weshalb diese Intervention nur in erfahrenen Zentren durchgeführt werden sollte.

\section{Lebertransplantation}

Das Vorliegen einer Pfortaderthrombose stellt heute nur noch selten eine Kontraindikation für eine Lebertransplantation dar, erhöht jedoch den technischen Anspruch an die Operation sowie die postoperative Morbidität und Mortalität [33]. Dies ist vor allem der Fall, wenn eine Pfortaderthrombose erst zum Zeitpunkt der Transplantation festgestellt wird. 
Tab. 3. Stadieneinteilung der hepatischen Enzephalopathie nach den WestHaven-Kriterien

\begin{tabular}{|c|c|c|c|}
\hline Stadium & Bewusstseinszustand & Verhalten & Neurologische Befunde \\
\hline 0 (subklinisch) & normal & normal & $\begin{array}{l}\text { unauffällig (auffällige } \\
\text { psychometrische Tests) }\end{array}$ \\
\hline 1 & $\begin{array}{l}\text { leichte Aufmerksamkeitsstörung, } \\
\text { Wesensveränderungen }\end{array}$ & $\begin{array}{l}\text { Konzentrationsstörungen, } \\
\text { leichte Verwirrung }\end{array}$ & $\begin{array}{l}\text { Apraxie, eventuell «Flapping } \\
\text { Tremor» }\end{array}$ \\
\hline 2 & Lethargie & $\begin{array}{l}\text { Desorientiertheit, } \\
\text { Verhaltensauffälligkeiten }\end{array}$ & «Flapping Tremor», Dysarthrie \\
\hline 3 & Somnolenz & $\begin{array}{l}\text { schwere Desorientierung, } \\
\text { Aggressivität }\end{array}$ & $\begin{array}{l}\text { Myoklonien, Hyperreflexie, } \\
\text { eventuell positives } \\
\text { Babinski-Zeichen }\end{array}$ \\
\hline 4 & Koma & Koma & $\begin{array}{l}\text { unter anderem fehlende Reaktion } \\
\text { auf Schmerzreiz }\end{array}$ \\
\hline
\end{tabular}

Insbesondere bei Patienten auf der Warteliste zur Lebertransplantation sollte daher regelmäßig die Durchgängigkeit der Pfortader untersucht werden, um gegebenenfalls therapeutische Massnahmen (Antikoagulation oder TIPS) einzuleiten. Ziel dieser Massnahmen sollte sein, intraoperativ eine konventionelle End-zu-End-Anastomose der Pfortader zu ermöglichen.

\section{Hepatische Enzephalopathie}

Die hepatische Enzephalopathie tritt typischerweise bei Patienten mit fortgeschrittener Leberzirrhose auf und ist als potenziell reversible neuropsychiatrische Störung zu betrachten [34]. Die klinische Symptomatik ist vielfältig und reicht von geringsten neurologischen Veränderungen hin zum Coma hepaticum. Ammoniak ist eine wesentliche toxische Substanz als möglicher Auslöser für eine hepatische Enzephalopathie. Veränderungen der Blut-Hirn-Schranke, fehlerhafte Neurotransmission und Störungen des GABAergen und Benzodiazepin-Metabolismus spielen jedoch auch eine mögliche Rolle. Die Identifizierung und Behandlung der auslösenden Faktoren (Tab. 2) stellen die wesentliche Behandlungsgrundlage der hepatischen Enzephalopathie dar.

\section{Diagnostik}

Mit Ausnahme des «Flapping Tremor» (asterixis) weist die hepatische Enzephalopathie keine spezifischen Symptome auf. Neurologische und psychiatrische Veränderungen gemäss der Stadieneinteilung nach den West-Haven-Kriterien sind in Tabelle 3 zusammengefasst. Frühe Stadien (subklinische Enzephalopathie und Stadium 1) können mit psychometrischen Tests oder einer Messung der kritischen Flimmerfrequenz detektiert werden [35, 36]. Fokal-neurologische Symptome oder ein sehr rasch eintretendes Koma ohne Prodromi sind hingegen untypisch und sollten Anlass zu einer weiterführenden Diagnostik mittels kranialer Computertomographie und gegebenenfalls Liquorpunktion geben. Eine zerebrale Bildgebung ist zudem im Stadium 4 zum Ausschluss eines erhöhten Hirndrucks bzw. einer intrakraniellen Blutung erforderlich.

Die venöse Ammoniakkonzentration korreliert unzureichend mit dem Grad der hepatischen Enzephalopathie [37] und spielt auch wegen der zahlreichen Fehlermöglichkeiten in der Präanalytik keine Rolle für die Akutdiagnostik der hepatischen Enzephalopathie. Zudem erlaubt die venöse Ammoniakkonzentration keine Prognose über den klinischen Verlauf [38] und ist daher für die Akutdiagnostik von Patienten mit hepatischer Enzephalopathie verzichtbar. Vielmehr sollte bei klinisch manifester Enzephalopathie die Diagnostik bereits auf mögliche, in Tabelle 1 genannte Auslöser fokussieren: Serumelektrolyte und Nierenretentionswerte, Blutbild und CRP, Blutkulturen, diagnostische Aszitespunktion zum Ausschluss einer spontanen bakteriellen Peritonitis, UrinStix, Urin-Sediment und gegebenenfalls Urin-Drogenscreening sowie eine Röntgen-Thoraxaufnahme sollten veranlasst werden.

\section{Therapie}

$\mathrm{Zu}$ den wesentlichen supportiven Massnahmen bei Patienten mit hepatischer Enzephalopathie gehört die Identifikation und Behandlung der auslösenden Faktoren (siehe Tab. 1). Bei komatösen Patienten (Stadium 3 und 4) sollte die Verlegung auf eine Intensivstation erfolgen und - zumindest im Stadium 4 - in Abhängigkeit von der Gesamtprognose des Patienten die Schutzintubation diskutiert werden.

\section{Nichtresorbierbare Disaccharide}

Lactulose und Lactitol werden bei hepatischer Enzephalopathie seit Langem mit Erfolg eingesetzt. Die Dosierung sollte so gewählt werden, dass 2-3 weiche Stuhlgänge pro Tag induziert werden. Die Wirkung der Disaccharide wird durch folgende Mechanismen erklärt:

- Beschleunigung der Darmtransitzeit und somit verminderte Ammoniakresorption; 
- Absenkung des pH-Werts des Darminhalts, wodurch Ammoniak überwiegend in ionisierter Form vorliegt und schlechter resorbiert wird;

- Reduktion der intestinalen Ammoniakproduktion durch Hemmung der Glutaminaseaktivität.

Auch wenn eine aktuelle Metaanalyse [39] den Nutzen der Disaccharide infrage stellt, werden diese ubiquitär mit gutem Erfolg für die Therapie der hepatischen Enzephalopathie eingesetzt. Zudem zeigen neuere Studien die Effektivität dieser Substanzen [40].

\section{Antibiotika}

Unter der Vorstellung einer selektiven Elimination Ammoniak-produzierender Darmbakterien kommen Antibiotika insbesondere bei schweren bzw. therapierefraktären Enzephalopathieformen zum Einsatz.

Neomycin ist ein kaum resorbierbares Aminoglykosid, das lange Jahre in einer Dosierung von 4-6 g/Tag für die Therapie der hepatischen Enzephalopathie zum Einsatz kam. Geringe Mengen gelangen jedoch in die Zirkulation und können zu Nephrotoxizität und irreversibler Ototoxizität führen, weshalb sich ein längerfristiger Einsatz über mehr als 7 Tage verbietet.

Die klinische Wirksamkeit von Rifaximin, einem synthetischen Derivat des Rifamycin, konnte in mehreren klinischen Studien gezeigt werden [41]. Es wird nur zu einem sehr geringen Anteil resorbiert und hat praktisch keine Nebenwirkungen. Gegen eine Langzeittherapie der hepatischen Enzephalopathie mit Rifaximin sprechen allerdings der derzeit sehr hohe Preis sowie das hohe Risiko des Auftretens multiresistenter Keime.

\section{Flumazenil}

Der selektive Bezodiazepin-Rezeptorantagonist Flumazenil kann in einzelnen Fällen kurzfristig zu einer signifikanten Besserung der Enzephalopathie führen. Insgesamt konnte in zahlreichen Studien jedoch kein Effekt hinsichtlich einer Besserung der Enzephalopathie oder Mortalität gezeigt werden, weshalb von einem routinemäßigen klinischen Einsatz abgeraten wird [42].

\section{Sonstige Maßnahmen}

Bei therapierefraktärer Enzephalopathie nach Anlage eines TIPS sollte die interventionelle Reduktion des Shunt-Durchmessers bzw. der TIPS-Verschluss erwogen werden [43].

Die früher übliche Eiweißrestriktion bei der hepatischen Enzephalopathie ist ohne belegten Vorteil, verschlechtert die Eiweißbilanz und sollte in der Regel unterlassen werden [44]. Unzureichende spontane Nahrungsaufnahme oder ärztlich verordnete Nahrungskarenz können eine Katabolie induzieren, die über einen erhöhten Abbau von Körpereiweiß die Ausbildung einer hepatischen Enzephalopathie begünstigt [45]. Eiweißexzesse sollten jedoch vermieden werden.

\section{Disclosure Statement}

A. Gerbes ist als Berater für CSV Behring, Marburg, tätig.

\section{Literatur}

1 Gerbes AL, Gülberg V, Sauerbruch T, et al.: S3Leitlinie Aszites, spontan bakterielle Peritonitis, hepatorenales Syndrom. Z Gastroenterol 2011;49: 749-779.

2 Abecasis R, Guevara M, Miguez C, et al.: Longterm efficacy of torasemide compared with frusemide in cirrhotic patients with ascites. Scand J Gastroenterol 2001;36:309-313.

3 Fiaccadori F, Pedretti G, Pasetti G, et al.: Torasemide versus furosemide in cirrhosis: a long-term, double-blind, randomized clinical study. Clin Investig 1993;71:579-584.

4 Gerbes AL, Bertheau-Reith U, Falkner C, et al.: Advantages of the new loop diuretic torasemide over furosemide in patients with cirrhosis and ascites. A randomized, double blind cross-over trial. J Hepatol 1993;17:353-358.

5 Arroyo V, Gines P, Gerbes AL, et al.: Definition and diagnostic criteria of refractory ascites and hepatorenal syndrome in cirrhosis. International Ascites Club. Hepatology 1996;23:164-176.

6 Rosemurgy AS, Zervos EE, Clark WC, et al.: TIPS versus peritoneovenous shunt in the treatment of medically intractable ascites: a prospective randomized trial. Ann Surg 2004;239:883-889; discussion 889-891.
7 Bellot B, Welker M, Soriano G, et al.: Description of an automated low-flow ascites (alfa) pump system for the treatment of refractory ascites in patients with cirrhosis. Preliminary report. J Hepatol 2012;56:S23

8 Rossle M, Gerbes AL: TIPS for the treatment of refractory ascites, hepatorenal syndrome and hepatic hydrothorax: a critical update. Gut 2010;59: 988-1000.

9 Wong F, Sniderman K, Liu P, et al.: Transjugular intrahepatic portosystemic stent shunt: effects on hemodynamics and sodium homeostasis in cirrhosis and refractory ascites. Ann Intern Med 1995; 122:816-822.

10 Gines P, Uriz J, Calahorra B, et al.: Transjugular intrahepatic portosystemic shunting versus paracentesis plus albumin for refractory ascites in cirrhosis. Gastroenterology 2002;123:1839-1847.

11 Rössle M, Ochs A, Gülberg V, et al.: A comparison of paracentesis and transjugular intrahepatic portosystemic shunting in patients with ascites. N Engl J Med 2000;342:1701-1707.

12 Salerno F, Camma C, Enea M, et al.: Transjugular intrahepatic portosystemic shunt for refractory ascites: a meta-analysis of individual patient data. Gastroenterology 2007;133:825-834.
13 Sanyal AJ, Genning C, Reddy KR, et al.: The North American Study for the Treatment of Refractory Ascites. Gastroenterology 2003;124:634-641.

14 Salerno F, Merli M, Riggio O, et al.: Randomized controlled study of TIPS versus paracentesis plus albumin in cirrhosis with severe ascites. Hepatology 2004;40:629-635.

15 Gülberg V, Liss I, Bilzer M, et al.: Improved quality of life in patients with refractory or recidivant ascites after insertion of transjugular intrahepatic portosystemic shunts. Digestion 2002;66:127-130.

16 Salerno F, Gerbes A, Gines P, et al.: Diagnosis, prevention and treatment of hepatorenal syndrome in cirrhosis. Gut 2007;56:1310-1318.

17 Gluud LL, Christensen K, Christensen E, Krag A: Systematic review of randomized trials on vasoconstrictor drugs for hepatorenal syndrome. Hepatology 2010;51:576-584.

18 Wong F, Raina N, Richardson R: Molecular adsorbent recirculating system is ineffective in the management of type 1 hepatorenal syndrome in patients with cirrhosis with ascites who have failed vasoconstrictor treatment. Gut 2010;59:381-386.

19 Acosta S, Alhadad A, Verbaan H, Ogren M: The clinical importance in differentiating portal from mesenteric venous thrombosis. Int Angiol 2011;30: 71-78. 
20 Rajani R, Björnsson E, Bergquist A, et al.: The epidemiology and clinical features of portal vein thrombosis: a multicentre study. Aliment Pharmacol Ther 2010;32:1154-1162.

21 Amitrano L, Brancaccio V, Guardascione MA, et al.: Portal vein thrombosis after variceal endoscopic sclerotherapy in cirrhotic patients: role of genetic thrombophilia. Endoscopy 2002;34:535-538.

22 Francoz C, Belghiti J, Vilgrain V, et al.: Splanchnic vein thrombosis in candidates for liver transplantation: usefulness of screening and anticoagulation. Gut 2005;54:691-697.

23 Zocco MA, Di Stasio E, De Cristofaro R, et al.: Thrombotic risk factors in patients with liver cirrhosis: correlation with MELD scoring system and portal vein thrombosis development. J Hepatol 2009;51:682-689.

24 Amitrano L, Brancaccio V, Guardascione MA, et al.: Inherited coagulation disorders in cirrhotic patients with portal vein thrombosis. Hepatology 2000;31:345-348.

25 DeLeve LD, Valla DC, Garcia-Tsao G; American Association for the Study Liver Diseases: AASLD Practice Guidelines: Vascular disorders of the liver. Hepatology 2009;49:1729-1764.

26 Tublin ME, Dodd GD 3rd, Baron RL: Benign and malignant portal vein thrombosis: differentiation by CT characteristics. AJR Am J Roentgenol 1997; 168:719-723.

27 de Franchis R; Baveno V Faculty: Revising consensus in portal hypertension: report of the Baveno $\mathrm{V}$ consensus workshop on methodology of diagnosis and therapy in portal hypertension. J Hepatol 2010; 53:762-768.
28 Amitrano L, Guardascione MA, Menchise A, et al.: Safety and efficacy of anticoagulation therapy with low molecular weight heparin for portal vein thrombosis in patients with liver cirrhosis. J Clin Gastroenterol 2010;44:448-451.

29 Han G, Qi X, He C, et al.: Transjugular intrahepatic portosystemic shunt for portal vein thrombosis with symptomatic portal hypertension in liver cirrhosis. J Hepatol 2011;54:78-88.

30 Luca A, Miraglia R, Caruso S, et al.: Short- and long-term effects of the transjugular intrahepatic portosystemic shunt on portal vein thrombosis in patients with cirrhosis. Gut 2011;60:846-852.

31 Senzolo M, Sartori T, Rossetto V, et al.: Prospective evaluation of anticoagulation and transjugular intrahepatic portosistemic shunt for the management of portal vein thrombosis in cirrhosis. Liver Int 2012;32:919-927.

32 D'Avola D, Bilbao JI, Zozaya G, et al.: Efficacy of TIPS for the prevention of total portal vein thrombosis in cirrhotic patients waiting for liver transplantation. J Hepatol 2012;56(suppl 2):S81 (abstract).

33 Francoz C, Valla D, Durand F: Portal vein thrombosis, cirrhosis, and liver transplantation. J Hepatol 2012;57:203-212.

34 Bismuth M, Funakoshi N, Cadranel JF, Blanc P. Hepatic encephalopathy: from pathophysiology to therapeutic management. Eur J Gastroenterol Hepatol 2011;23:8-22.

35 Montagnese S, Amodio P, Morgan MY: Methods for diagnosing hepatic encephalopathy in patients with cirrhosis: a multidimensional approach. Metab Brain Dis 2004:19:281-312.
36 Häussinger D: Hepatic encephalopathy. Acta Gastroenterol Belg 2010;73:457-464.

37 Tarantino G, Citro V, Esposito P, et al.: Blood ammonia levels in liver cirrhosis: a clue for the presence of portosystemic collateral veins. BMC Gastroenterol 2009;9:21.

38 Bajaj JS: Review article: The modern management of hepatic encephalopathy. Aliment Pharmacol Ther 2010;31:537-547.

39 Als-Nielsen B, Gluud LL, Gluud C: Nonabsorbable disaccharides for hepatic encephalopathy. Cochrane Database Syst Rev 2004;2:CD003044.

40 Prasad S, Dhiman RK, Duseja A, et al.: Lactulose improves cognitive functions and health-related quality of life in patients with cirrhosis who have minimal hepatic encephalopathy. Hepatology 2007; 45:549-559.

41 Rivkin A, Gim S: Rifaximin: new therapeutic indication and future directions. Clin Ther 2011;33: 812-827.

42 Als-Nielsen B, Gluud LL, Gluud C: Benzodiazepine receptor antagonists for hepatic encephalopathy. Cochrane Database Syst Rev 2004:2:CD002798.

43 Gerbes AL, Waggershauser T, Holl J, et al.: Experiences with novel techniques for reduction of stent flow in transjugular intrahepatic portosystemic shunts. Z Gastroenterol 1998;36:373-377.

44 Córdoba J, López-Hellín J, Planas M, et al.: Normal protein diet for episodic hepatic encephalopathy: results of a randomized study. J Hepatol 2004;41:38-43.

45 Zillikens MC, van den Berg JW, Wattimena JL, et al.: Nocturnal oral glucose supplementation. The effects on protein metabolism in cirrhotic patients and in healthy controls. J Hepatol 1993;17:377-383. 